\title{
Editorial
}

\section{Advanced Cloud Computing and Novel Applications}

\author{
Bao Rong Chang, ${ }^{1}$ Ngoc Thanh Nguyen, ${ }^{2}$ Bay Vo, ${ }^{3}$ and Hui-Huang Hsu ${ }^{4}$ \\ ${ }^{1}$ Department of Computer Science \& Information Engineering, National University of Kaohsiung, Kaohsiung 81148, Taiwan \\ ${ }^{2}$ Institute of Informatics, Wroclaw University of Technology, 50370 Wroclaw, Poland \\ ${ }^{3}$ Faculty of Information Technology, Ton Duc Thang University, Ho Chi Minh City 70000, Vietnam \\ ${ }^{4}$ Department of Computer Science \& Information Engineering, Tamkang University, New Taipei City 25137, Taiwan
}

Correspondence should be addressed to Bao Rong Chang; brchang@nuk.edu.tw

Received 13 July 2015; Accepted 22 July 2015

Copyright ( 2015 Bao Rong Chang et al. This is an open access article distributed under the Creative Commons Attribution License, which permits unrestricted use, distribution, and reproduction in any medium, provided the original work is properly cited.

The study on advanced cloud computing encompasses a large number of the most important as well as promising direction for scientific research and development in the era of advanced novel applications which explores the fundamental roles and interactions as well as practical impacts of advanced computing and information technologies on the next generation of cloud-empowered products, systems, services, and activities. Advanced information technology and computing engineering have involved internet of things, ubiquitous computing, social networks, and data/knowledge grids, as well as cloud computing and service-oriented architecture.

A lot of relevant topics have been raised in this issue, though many other topics could be concerned. This special issue is intended to foster a high profile, leading edge forum for people, either in the scope of R\&D projects, engineering, or business applications, who are working in the field to contribute and disseminate innovative new work on advanced cloud computing. Here high quality technical papers in all aspects of advanced cloud computing including emerging trends and applications, theoretical studies, and experimental prototypes have been presented.

Z. Peng et al. deal with cloud computing resources provisioning based on reinforcement learning. With the introduction of the concepts of segmentation service level agreement (SSLA) and utilization unit time cost (UUTC), this study designed a novel optimization object function and employed reinforcement learning to solve resources provisioning. L. Pan and D. Wang develop metaheuristic solutions based on the genetic algorithm to tickle IaaS cloud provider revenue maximization (ICPRM) problem. The paper proposed by S. Li et al. gives a novel multilevel data encoding scheme satisfying these requirements in mobile cloud computing applications, particularly in the field of telerehabilitation.

The security management is addressed in three aspects. K. Mazur et al. implemented the role-based access control method in quality of protection modeling language (QoP-ML) and evaluated its performance in terms of mentioned factors to determine the most secure, energy-efficient, environmental-friendly security mechanisms. In cloud computing people really care user data protection through identity management; L. Wu et al. introduce a reputation mechanism and design a reputation-based identity management model for cloud computing to solve these problems. In order to guarantee the credibility of this information, D. Wang et al. present a novel trust degree model of the credibility information and then propose a new composite semantic web service selection approach based on this credible information.

Besides those, there are several interesting topics in the issue. C.-J. Tang and M.-R. Dai suggest modeling and analysis of queueing-based vary-on/vary-off (VOVO) schemes for server clusters. X. Liu et al. preform wireless-uplinks-based energy-efficient scheduling in mobile cloud computing. C.L. Chou et al. realize multicriteria resource brokering in cloud computing for streaming service. Y. Liu and W. Wei establish a replication-based mechanism for fault tolerance in MapReduce framework. 
By compiling these papers, we hope to enrich our readers and researchers with respect to the various problems and their solutions in cloud computing and novel applications.

Bao Rong Chang Ngoc Thanh Nguyen

Bay Vo

Hui-Huang Hsu 


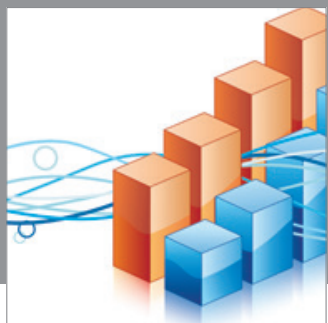

Advances in

Operations Research

mansans

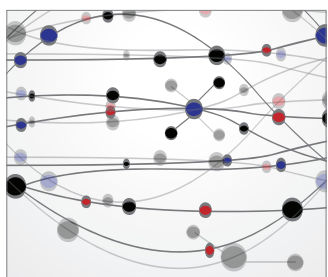

The Scientific World Journal
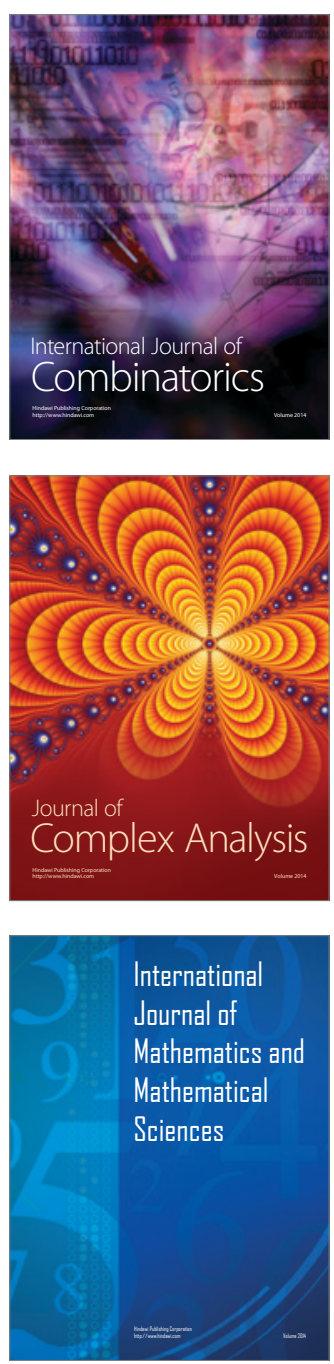
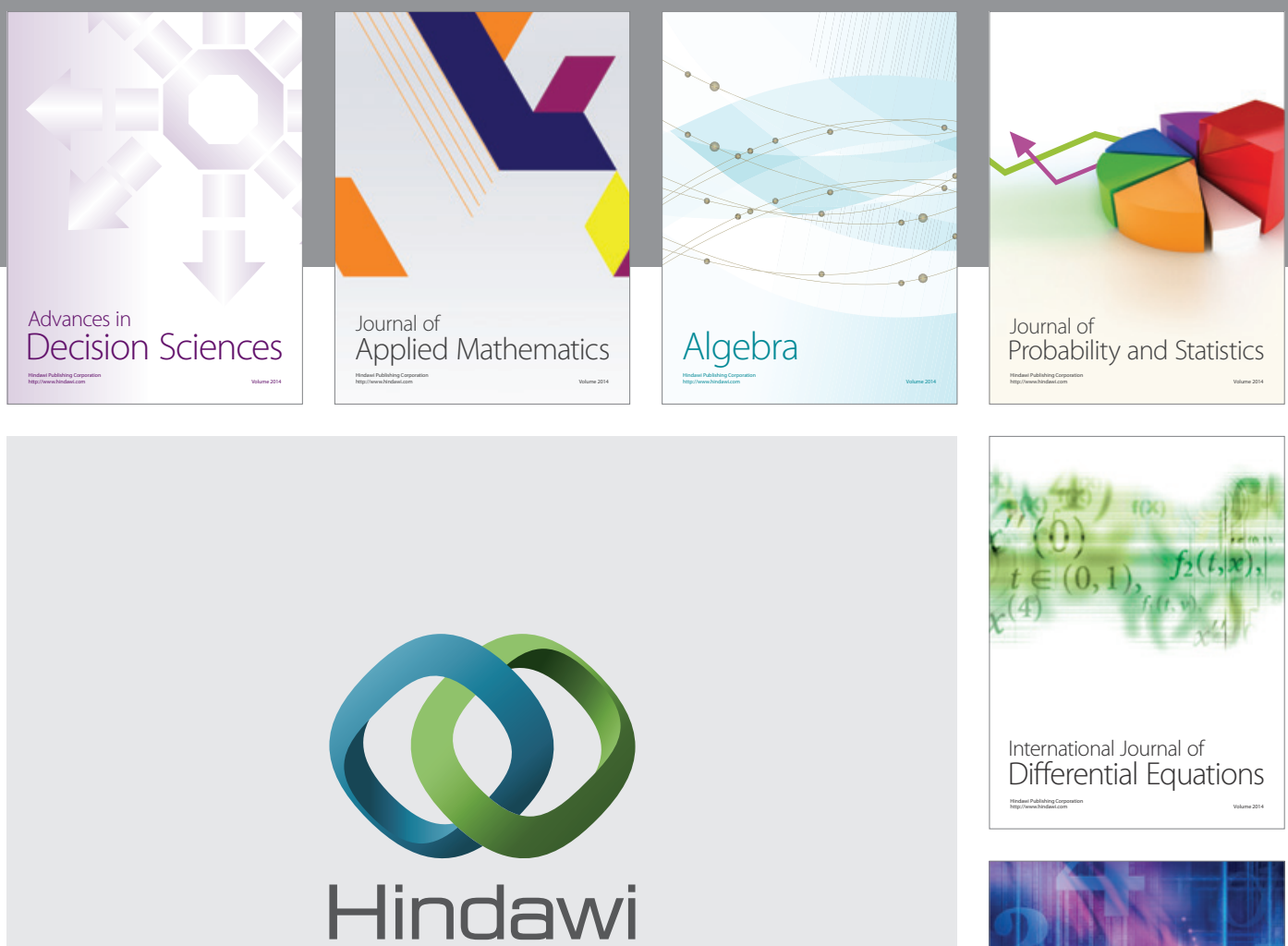

Submit your manuscripts at http://www.hindawi.com
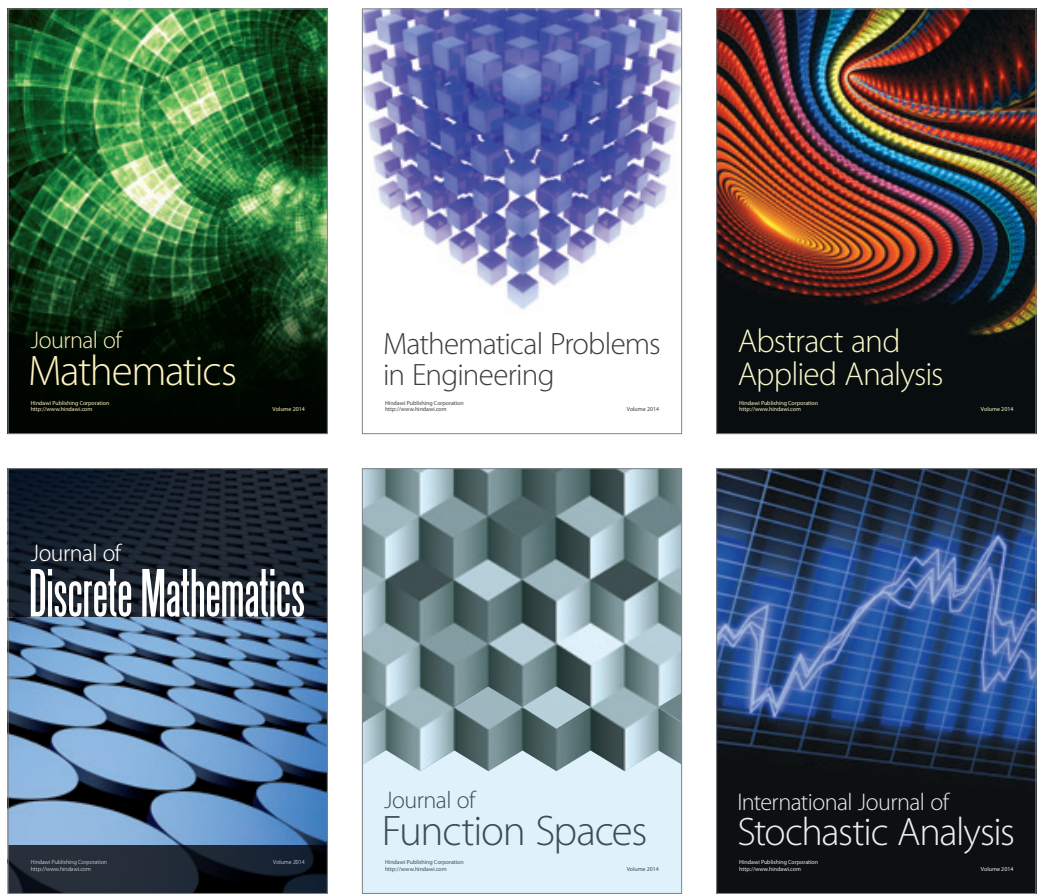

Journal of

Function Spaces

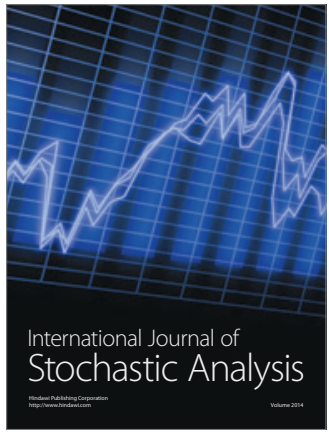

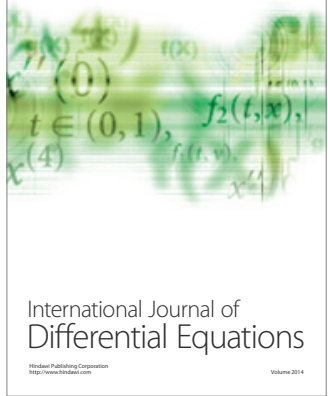
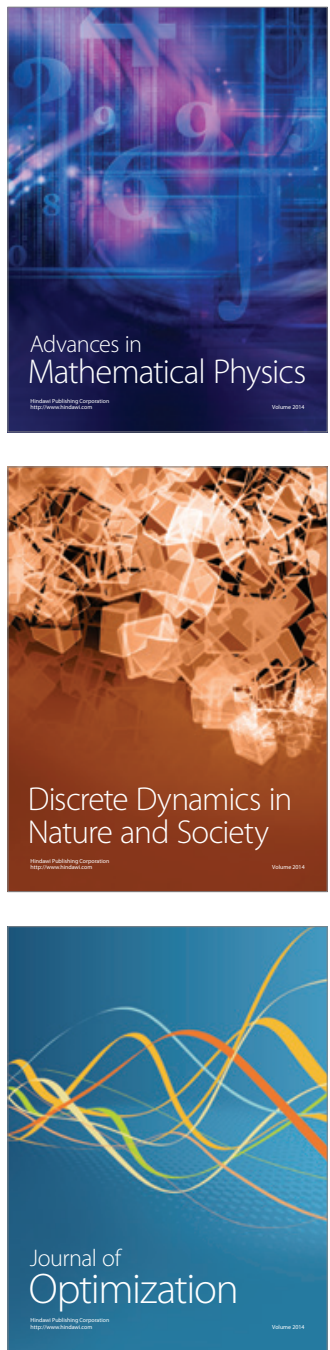